\title{
Dreaming of the future of stroke: translation of bench to bed
}

\section{Oh Young Bang ${ }^{1}$, Won Hyuk Chang ${ }^{2}$, Hong-Hee Won ${ }^{3}$}

${ }^{1}$ Department of Neurology, Samsung Medical Center, Sungkyunkwan University School of Medicine, Seoul, Korea

${ }^{2}$ Department of Physical and Rehabilitation Medicine, Samsung Medical Center, Sungkyunkwan University School of Medicine, Seoul, Korea

${ }^{3}$ Samsung Advanced Institute for Health Sciences and Technology (SAIHST), Samsung Medical Center, Sungkyunkwan University School of Medicine, Seoul, Korea

Received: August 10, 2017

Revised: August 30, 2017

Accepted: September 1, 2017

Corresponding author:

Oh Young Bang

Department of Neurology,

Samsung Medical Center,

Sungkyunkwan University

School of Medicine, 81 Irwon-

ro, Gangnam-gu, Seoul 06351,

Korea

Tel: +82-2-3410-3599

E-mail:

ohyoung.bang@samsung.com

\section{ABSTRACT}

Stroke is one of the leading causes of death and the most common cause of disability in adults. Remarkable advances in stroke research have been achieved during the past decades. Stroke fatalities have decreased significantly worldwide owing to improved stroke care because of increased public awareness of stroke symptoms, improved acute stroke therapy, and improved stroke prevention approaches. There are currently significant developments in new technologies with the potential to be used daily in clinical practice in patients with stroke. In this review, we have selected and discussed some of the key areas related to stroke, namely big data, artificial intelligence, precision medicine, medical devices, and stem cells.

Keywords: Artificial intelligence; Big data; Precision medicine; Stem cells; Stroke
This is an Open Access article distributed under the terms of the Creative Commons Attribution Non-Commercial License (http:// creativecommons.org/licenses/ by-nc/4.0/).

\section{INTRODUCTION}

During the past decades, remarkable advances have been achieved in stroke research. In addition, there have been significant progresses in new technologies that could potentially be used in everyday medical practices in patients with stroke. Indeed, the technological development enabled not only changes in our daily lives but also many changes in medical care. In the past, advances in the medical field were important for the diagnosis and treatment of diseases. The recent notable technological development in non-medical fields has made it important to apply these recent progresses to the medial field and healthcare. With personal computers becoming more affordable and popular, information and communication technology are revolutionized. Similarly, the plummeting costs of genome sequencing have transformed biomedical research and clinical practice. The President of the United States, Barack Obama, called for the development of the 'All of Us Research Program' (formerly known as the 'Precision Medicine Initiative Program') in January 2015. In addition, stem cell research is currently one of the most exciting and fast-paced areas of biomedical research and development. Every year, the Massa- 
chusetts Institute of Technology and other leading institutions select 10 to 20 areas of future medicine. In this review, we have selected and discussed some of the key areas in stroke research.

\section{SEARCH STRATEGIES AND SELECTION CRITERIA}

We searched PubMed to identify research articles in English published till June 2017. The following search terms were used: "stroke," "cerebral infarction," "future medicine," "precision medicine," "artificial intelligence," "big data," and "stem cells." Additionally, we searched the references of selected relevant articles and reviews. The final reference list was generated on the basis of originality and relevance to the review's topic. Because of space limitations, individual technologies were not discussed in detail.

\section{HISTORY OF MAJOR DISCOVERIES IN STROKE RESEARCH}

To date, there have been several remarkable new developments in stroke research (Fig. 1). With advances in neuroimaging and other diagnostic techniques, the diagnosis of stroke, etiological evaluations and quantitation of the ischemic zone have improved significantly [1,2]. Several new medications, surgical techniques, and other interventions were introduced and proved their efficacy in managing stroke. Furthermore, there has been a notable decline in the stroke incidence worldwide owing to the advancements in the control of traditional stroke risk factors, and to the improved identification of non-traditional risk factors of stroke [3].

The historical advances in the stroke field comprise previous efforts to segment patients with stroke. There is no 'one-sizefits-all' treatment option for patients with stroke. A precise classification of stroke is important because its etiological mechanisms are far more diverse than those of other diseases such as coronary heart diseases. In addition, the individual characterization of patients with stroke is primordial in managing the disease because the mechanisms of ischemic brain death and the ischemic zone vary greatly among patients. Indeed, the treatment of choice and the response to stroke therapies may be greatly influenced by such factors. The selection of optimal candidate patients has been considered in every strategy of stroke treatment, such as a time window of 3 to 4.5 hours for intravenous thrombolysis with tissue plasminogen activator [4,5], the presence of large vessel occlusion but small core with good collaterals for endovascular therapy [6-10], the degree of stenosis and instability of plaque for carotid revascularization [11,12], aggressiveness of antiplatelet

Efforts for precision of stroke patients Major discoveries in history

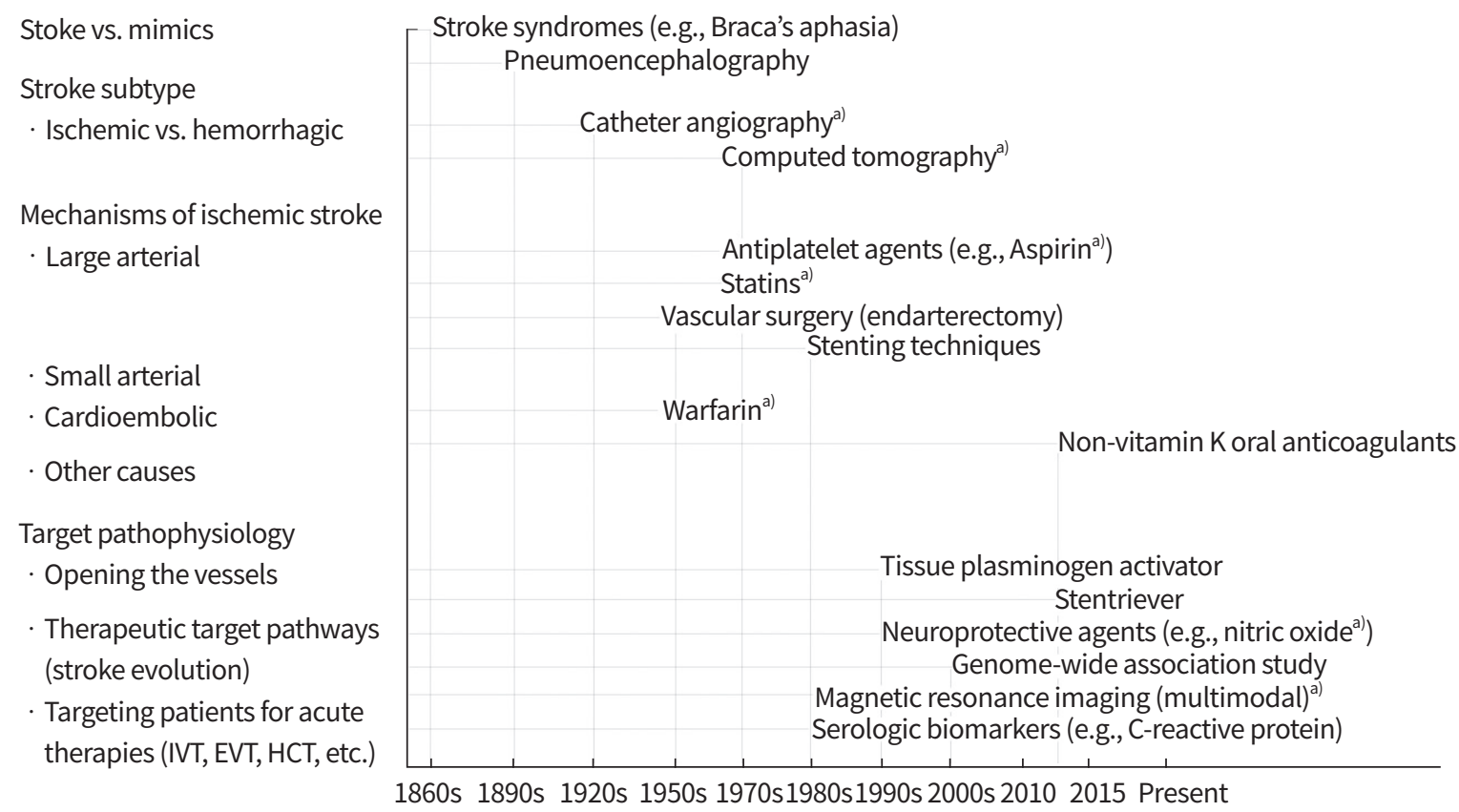

Fig. 1. History of major discoveries in the diagnosis and management of stroke. IVT, intravenous thrombolysis; EVT, endovascular therapy; HCT, hemicraniectomy. ${ }^{\text {a) }}$ The Nobel prize awarded. 
agents and statins for non-cardioembolic stroke [13-15], and risk schemes for the use of oral anticoagulants to prevent cardioembolic stroke (e.g., CHA2DS2-VASc score).

\section{RECENT ADVANCES IN FUTURE MEDI- CINE IN THE STROKE FIELD}

In this review, we selected five key emerging areas as the future stroke medicine, namely big data, precision medicine, artificial intelligence, medical devices, and stem cells. Indeed, these areas have recently gained increasing focus and have being extensively studied and applied in clinical management of stroke. Although reflecting different entities, these areas are not independent of each other because when one of those develops, the other areas merge and evolve synergistically (Fig. 2).

These key focus areas of future medicine are particularly helpful in the field of stroke, probably owing to the following characteristics of stroke compared to other medical illnesses. First, patients with stroke exhibit various clinical phenotypes and prognosis, making the diagnosis and clinical prediction difficult (heterogeneity). Second, stroke is associated with various risk factors, both genetic and environmental, which should be considered in stroke prevention (multifactorial). Third, the pathophysiology and outcome of stroke are very complex; thereby, necessitating integrative analysis of various causative and surrogate biomarkers, i.e., molecular and imaging studies (multi-level data). Fourth, in the treatment process, the communication between health service provid- ers and patients is more important than unilateral education (bilateral communication). Fifth, stroke is a very common disease, public health plays an important role in the prevention and management of stroke, and related patient information is being accumulated at a large scale (publicity). Finally, for patients with severe neurological deficits, novel therapeutic strategies are needed (intractability).

\section{Big data}

Evidence from randomized controlled trials (RCTs) forms the basis for national and international guidelines for the management of patients with stroke. However, abundant and strict enrollment criteria may limit the validity and applicability of the results of RCTs to clinical practice. Recent studies of antiplatelet therapy for non-cardioembolic stroke, non-vitamin $\mathrm{K}$ oral anticoagulants for non-valvular atrial fibrillation, and surgical treatment for intracranial hemorrhage showed that patients with stroke enrolled in RCTs are only partially representative of patients in clinical practice [16-18]. Big data can be useful for confirming the results of RCTs in the real world. For example, the blood pressure target supported by the results of a recent RCT (Systolic Blood Pressure Intervention Trial [SPRINT], systolic blood pressure $<120$ $\mathrm{mm} \mathrm{Hg}$ ) [19] is substantially lower than that recommended in the 2014 hypertension guidelines developed by the panel appointed to the 8 th Joint Committee $(150 / 90 \mathrm{~mm} \mathrm{Hg}$ for patients $\geq 60$ years and $140 / 90 \mathrm{~mm} \mathrm{Hg}$ for younger patients or patients with diabetes or chronic kidney disease) [20]. A big data approach using a nationally representative sample in

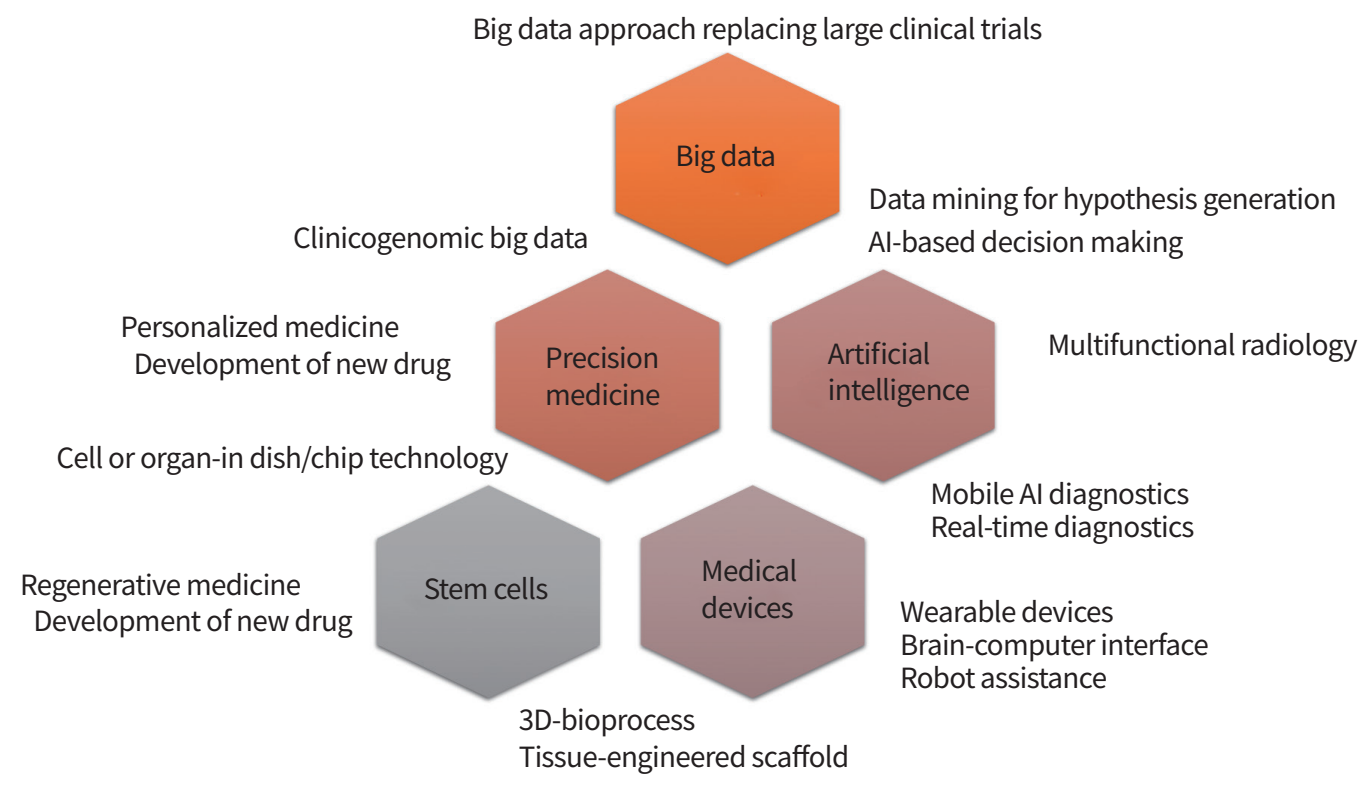

Fig. 2. Five key areas of future medicine and related research field in stroke. Al, artificial intelligence; 3D, three-dimensional. 
Korea showed that the proportion of adults with hypertension satisfying the respective goals would decrease from $70.8 \%$ under the 2014 guidelines to $11.9 \%$ under the SPRINT criteria. Furthermore, the 10 -year predicted risk of cardiovascular events differed significantly as well, and corresponded to $6.15 \%$ in patients who met the SPRINT goals and $7.65 \%$ in those who met the 2014 recommendation goals [21]. In addition, the application of existing big data, such as national health insurance data, can answer new questions in stroke research, such as those related to vascular dementia research [22]. However, it should be noted that bigger data does not necessarily mean better data because it may have inherent limitations and biases. The value of big data will be guaranteed by appropriate and efficient analytical methods.

\section{Artificial intelligence}

Data mining, also called knowledge discovery process, is the computing process of discovering patterns in large datasets involving methods at the intersection of machine learning, statistics, and database systems. In addition to the knowledge and experience of clinicians and the results of imaging studies, the accuracy and efficiency of prediction, diagnosis/ classification, prognosis, and management of stroke can be improved by the application of data mining techniques [2326]. Li and colleagues [24] identified predictive risk factors for ischemic stroke/thromboembolism in atrial fibrillation by using integrated machine learning and data mining approaches. They showed that this approach can achieve a higher prediction performance than previous prediction models for atrial fibrillation (such as $\mathrm{CHA}_{2} \mathrm{DS}_{2}$-VASc) [24]. Yoon and Gutierrez [26] showed that data mining is promising to discover new factors associated with post-stroke disability, such as exercise, employment and satisfaction of life. Data sets could be not only clinical data but also omics (i.e., genomics, transcriptomics, proteomics, and metabolomics) and neuroimaging data.

Understanding brain function is essential for predicting functional outcome, investigating recovery mechanisms, and promoting post-stroke functional recovery. So far, the function of the human brain has been explored from a network perspective with variable advanced neuroimaging techniques such as functional magnetic resonance image, transcranial magnetic stimulation, electroencephalography, magnetoencephalography, and near-infrared spectroscopy $[27,28]$. In this aspect, big data can be obtained in a relatively few patients with stroke with multimodal structural and functional neuroimaging technique. Kuceyeski et al. [29] re- ported that models of complex functions provided new insights into brain-behavior relationships through machine learning techniques with big data set of advanced neuroimaging techniques in 41 ischemic stroke patients. However, further studies with big data sets of functional neuroimaging from a larger number of patients with stroke are still needed for clinical application to patients with heterogeneity.

Machine learning is a subset of artificial intelligence. Machine learning techniques such as medical imaging, bioinformatics, and public health have recently been applied in healthcare. The application of artificial intelligence in stroke research is increasing, particularly in the prediction of stroke (e.g., risk factors [30] or fine particulate matter [PM2.5] [31]) and pervasive health monitoring using smart monitoring devices embedded in the living environment (e.g., real-time monitoring with smartphone for adherence of oral anticoagulants) [32]. Artificial intelligence can be particularly helpful in decision making in every step of endovascular therapy for acute ischemic stroke, including (1) clinical and imaging recognition of acute ischemic stroke in the ambulance or emergency room [33], and (2) outcome prediction after endovascular therapy [34]. In addition, pathological brain detection systems are being developed using magnetic resonance imaging and aim to assist neuroradiologists to make decisions in the management of neurologic diseases [35].

It was estimated that the amount of medical information available has been doubling every 5 years, and that only a small percentage of the knowledge that physicians use to make diagnosis and treatment decisions is evidence-based. In this context, artificial intelligence may assist physicians, including vascular neurologists, in everyday medical decision-making. The cognitive system IBM watson (IBM, Yorktown Heights, NY, USA) has the capacity to read 40 million documents in 15 seconds, and its efficacy in assisting oncologists in treating cancer patients is currently being tested.

\section{Medical devices}

Mobile medical devices were used to measure vital signs, heart rhythm, sleep pattern, and oxygen saturation, and to monitor the patients' behavior. Wearable devices that measure easily quantifiable data have been or are being developed to monitor in real time the patients' compliance to treatment or the status of risk-factor control. In patients with embolic stroke of unknown source, prolonged monitoring of the cardiac rhythm with implantable loop recorder or ambulatory electrocardiographic monitoring significantly improved the detection of atrial fibrillation, thereby enabling 
timely anticoagulation therapy $[36,37]$. In the near future, wearable sensors (i.e., skin or cloth-like structure) could replace such invasive and burdensome devices. Digital contact lens for measuring glucose levels from tears (patented by Google) could substitute blood sampling through venipuncture. Various data from the body fluid can be obtained and measured by point-of-care method and can be transferred to physicians.

Advancements in robotics and neuroscience can promote the improvement of functional impairment in patients with stroke through therapeutic assisting robots [38]. Therapeutic robot can improve the effects of stroke rehabilitation to provide more intensive, longer, and more repetitive tasks with more challenging and motivating components. In addition, the assisting robot can substitute the physical impairments in stroke patients by providing the mechanical power with a multi-sensor system [39,40].

Brain-computer interfaces ( $\mathrm{BCls}$ ) can connect a human brain and an external device without the need for human sensory and motor system [41]. Because $\mathrm{BCl}$ can substitute physical impairments such as motor and language functions, it enables patients with stroke who exhibit motor impairments to control the electrical device, which could be a computer cursor and a wheelchair [42]. Furthermore, $\mathrm{BCl}$ has been applied for motor neuromodulation purposes to induce real-time feedback to patients with stroke and to reward consistent production of neural features concordant with the motor function [41,43].

The medical applications for three-dimensional (3D) printing are expanding rapidly and are expected to revolutionize the healthcare system [44]. A high level of design and manufacturing personalization of 3D printing can remedy the shortcomings of the current orthoses in patients with stroke [45]. Nevertheless, many trials with 3D printing are anticipated for patients with stroke.

\section{Precision medicine}

Precision medicine relies on biomarkers, and enables a better classification of patients with their disease risk, prognosis, and treatment response. Identification of causative genetic variants may provide new avenues for stroke prevention (pre-stroke) and new therapeutic targets (post-stroke).

Four types of genetic studies have been conducted in stroke research: (1) single-gene mutation for rare stroke syndromes such as cerebral autosomal dominant arteriopathy with subcortical infarcts and leukoencephalopathy (CADASIL); (2) genome-wide association studies (GWAS), which identified common variants that are more frequent in patients with specific subtypes of stroke than in healthy individuals (case-control studies) or in responders for specific drugs than non-responders (pharmacogenomics); thus, providing GWAS-based genetic risk scores; (3) whole exome or genome DNA sequencing, which identified rare causal variants that may provide novel therapeutic targets and guidelines for stroke diagnosis and medication; and (4) other multi-omics studies (epigenome, transcriptome, proteome, metabolome, etc.), such as gene expression profiling to differentiate diverse stroke etiologies and clinical outcomes [46-50].

The scientific statement of the American Heart Association summarized four areas that have been translated into clinical practice, including cardiovascular disease risk prediction (still only modest evidence), pharmacogenomics (e.g., guide dosing of warfarin and clopidogrel), new drug development (e.g., proprotein convertase subtilisin/kexin type 9 [PCSK9] antagonists), and clinical actionability of genetic mutations (identifying individuals at risk) [51]. Genetic findings are particularly useful because they have recently been used to trigger drug development. A typical example is the monoclonal antibodies that inhibit PCSK9, which lowered the low density lipoprotein cholesterol and reduced the risk of stroke and cardiovascular events [52-54]. In addition, associations between genetic polymorphisms and functional outcomes have been reported in patients with stroke [55-59]. Brain-derived neurotrophic factor (BDNF) [55-57], apolipoprotein E [58], dopamine-related gene polymorphisms [59] have been proposed as genetic biomarkers to predict the functional outcomes in patients with stroke. In addition, the BDNF genotype is related to the different responses to non-invasive brain stimulation in patients with stroke [60]. Therefore, advancements in precision medicine could help design individual rehabilitation strategies for patients with stroke.

Notably, a recent, highly collaborative culture in genomic research has accelerated new discoveries in genomic studies worldwide [61]. In particular, the Global Alliance for Genomics and Health (http://genomicsandhealth.org), the 1000 Genomes Project (http://www.internationalgenome. org), ENCODE (ENCyclopedia Of DNA Elements, https:// www.encodeproject.org) Project, the Cancer Genome Atlas (TCGA, https://cancergenome.nih.gov), and UK Biobank cohort (http://www.ukbiobank.ac.uk) are representative examples of such efforts. Recent noticeable effort has been made to aggregate 123,136 whole exomes and 15,496 whole genomes in a publicly open database (gnomAD, genome Aggregate Database, http://gnomad.broadinstitute.org) that can 
be easily accessed to determine frequencies per population for variants of interest. The majority of such cohorts and databases offer public or controlled access to different levels (raw, pre-processed, analyzed, or summary statistics) of genomic and clinical data. Indeed, sharing and integrating large scale genome-phenome data will accelerate our understanding of the human complex diseases and the progress in human health.

\section{Stem cells and regenerative medicine}

Stem cell therapy is considered a potential regenerative strategy for patients with stroke who exhibit neurologic deficits. With the current advances in stem cell research and the understanding of their mechanisms of action, several clinical trials of stem cell therapy have been conducted in patients with stroke since 2005. These include studies using mesenchymal stem cells, bone marrow mononuclear cells, and neural stem/progenitor cells [62]. However, the results of these RCTs showed that adult stem cell therapy was safe but of modest efficacy; thereby, underscoring the need for new stem cell therapy strategies [63]. Several currently ongoing trials are examining the effects of allogeneic or manipulated stem cells in patients with stroke [64-66].

The primary hurdles for current stem cell therapies include the following: (1) the limited source of engraftable stem cells, (2) the presence of optimal time window for stem cell therapies, (3) the inherited limitation of the potential of adult stem cells, and (4) the possible transplanted cell-mediated adverse effects, such as tumor formation or stroke [63]. Several strategies have been revaluated in recent preclinical and clinical studies in order to overcome these hurdles, such as the off-the-shelf use of allogeneic stem cells to apply them at the acute phase of stroke, ex vivo manipulations of stem cells to enhance their efficacy, and cell-free paradigms using stem -cell derived extracellular vesicles/secretome.

The efficacy of stem cell therapy will be improved not only with the advances in the understanding of stem cell biology but also with the advances in techniques, such as biotechnology and bioengineering, to modulate the characteristics of stem cells. The 3D-bioprocess techniques are currently used for large-scale production of stem cells, while tissue-engineered scaffold could enhance stem cell survival $[67,68]$.

\section{CONCLUSION}

Stroke fatalities have decreased significantly worldwide owing to improved stroke care, including the public awareness of stroke symptoms and improvement in acute stroke therapy and in stroke prevention.

It is likely that application of future medicine, such as machine learning and precision medicine, will soon transform some sectors of the healthcare system. However, comparative studies on the effectiveness of these new techniques are not currently available, especially regarding the effects on health outcomes. The efficacy of these medical technologies has not yet been validated. More data are required to confirm the effects of application of precision and future medicine on the quality of health care. In addition, there is still a gap between bench and bed side. For future medicine to be applied on patients with stroke, clinicians need to be prepared to match the fast pace of technology developments. The Get with the Guidelines-Stroke registry, which evaluated 626,473 patients from 1,236 US hospitals, reported that electronic health records, which are an essential element in improving healthcare, were not associated with higher-quality care or better clinical outcomes in stroke [69]. Although there have been suggestions that machine learning incorporating multi-level data (genomics, omics, clinical, lifestyle, and environmental) will drive noticeable changes in health care within a few years, the introduction of machine learning technique in healthcare has not always been straightforward or without unintended and adverse effects [70]. There may be a problem in machine learning-based decision systems when no sufficient clinical context is represented. This is particularly important if physicians lose the awareness of the existence of clinical elements that are not included in the clinical record.

Nevertheless, the future favors the prepared mind.

\section{CONFLICTS OF INTEREST}

No potential conflict of interest relevant to this article was reported.

\section{ORCID}

Oh Young Bang https://orcid.org/0000-0002-7962-8751

\section{REFERENCES}

1. Bang OY, Ovbiagele B, Kim JS. Evaluation of cryptogenic stroke with advanced diagnostic techniques. Stroke 2014;45:1186-94.

2. Albers GW, Thijs VN, Wechsler L, Kemp S, Schlaug G, Ska- 
labrin E, et al. Magnetic resonance imaging profiles predict clinical response to early reperfusion: the diffusion and perfusion imaging evaluation for understanding stroke evolution (DEFUSE) study. Ann Neurol 2006;60:508-17.

3. Bang OY, Ovbiagele B, Kim JS. Nontraditional risk factors for ischemic stroke: an update. Stroke 2015;46:3571-8.

4. National Institute of Neurological Disorders and Stroke rt-PA Stroke Study Group. Tissue plasminogen activator for acute ischemic stroke. N Engl J Med 1995;333:1581-7.

5. Hacke W, Kaste M, Bluhmki E, Brozman M, Davalos A, Guidetti $D$, et al. Thrombolysis with alteplase 3 to 4.5 hours after acute ischemic stroke. N Engl J Med 2008;359:131729.

6. Berkhemer OA, Fransen PS, Beumer D, van den Berg LA, Lingsma HF, Yoo AJ, et al. A randomized trial of intraarterial treatment for acute ischemic stroke. N Engl J Med 2015;372:11-20.

7. Goyal M, Demchuk AM, Menon BK, Eesa M, Rempel JL, Thornton J, et al. Randomized assessment of rapid endovascular treatment of ischemic stroke. N Engl J Med 2015;372:1019-30.

8. Campbell BC, Mitchell PJ, Kleinig TJ, Dewey HM, Churilov L, Yassi N, et al. Endovascular therapy for ischemic stroke with perfusion-imaging selection. N Engl J Med 2015;372: 1009-18.

9. Saver JL, Goyal M, Bonafe A, Diener HC, Levy El, Pereira VM, et al. Stent-retriever thrombectomy after intravenous t-PA vs. t-PA alone in stroke. N Engl J Med 2015;372:228595.

10. Jovin TG, Chamorro A, Cobo E, de Miquel MA, Molina CA, Rovira A, et al. Thrombectomy within 8 hours after symptom onset in ischemic stroke. N Engl J Med 2015;372: 2296-306.

11. Barnett HJ, Taylor DW, Eliasziw M, Fox AJ, Ferguson GG, Haynes RB, et al. Benefit of carotid endarterectomy in patients with symptomatic moderate or severe stenosis. North American Symptomatic Carotid Endarterectomy Trial Collaborators. N Engl J Med 1998;339:1415-25.

12. Brott TG, Hobson RW 2nd, Howard G, Roubin GS, Clark WM, Brooks W, et al. Stenting versus endarterectomy for treatment of carotid-artery stenosis. N Engl J Med 2010; 363:11-23.

13. Wang Y, Wang Y, Zhao X, Liu L, Wang D, Wang C, et al. Clopidogrel with aspirin in acute minor stroke or transient ischemic attack. N Engl J Med 2013;369:11-9.

14. SPS3 Investigators, Benavente OR, Hart RG, McClure LA, Szychowski JM, Coffey CS, et al. Effects of clopidogrel added to aspirin in patients with recent lacunar stroke. N Engl J Med 2012;367:817-25.

15. Chimowitz MI, Lynn MJ, Derdeyn CP, Turan TN, Fiorella D, Lane BF, et al. Stenting versus aggressive medical therapy for intracranial arterial stenosis. N Engl J Med 2011; 365:993-1003.

16. Maasland L, van Oostenbrugge RJ, Franke CF, Scholte Op Reimer WJ, Koudstaal PJ, Dippel DW, et al. Patients enrolled in large randomized clinical trials of antiplatelet treatment for prevention after transient ischemic attack or ischemic stroke are not representative of patients in clinical practice: the Netherlands Stroke Survey. Stroke 2009;40:2662-8.

17. Yoon CH, Park YK, Kim SJ, Lee MJ, Ryoo S, Kim GM, et al. Eligibility and preference of new oral anticoagulants in patients with atrial fibrillation: comparison between patients with versus without stroke. Stroke 2014;45:2983-8.

18. Fonville AF, Samarasekera N, Hutchison A, Perry D, Roos YB, Al-Shahi Salman R. Eligibility for randomized trials of treatments specifically for intracerebral hemorrhage: community-based study. Stroke 2013;44:2729-34.

19. SPRINT Research Group, Wright JT Jr, Williamson JD, Whelton PK, Snyder JK, Sink KM, et al. A randomized trial of intensive versus standard blood-pressure control. N Engl J Med 2015;373:2103-16.

20. James PA, Oparil S, Carter BL, Cushman WC, Dennison-Himmelfarb C, Handler J, et al. 2014 Evidence-based guideline for the management of high blood pressure in adults: report from the panel members appointed to the Eighth Joint National Committee (JNC 8). JAMA 2014; 311:507-20.

21. Ko MJ, Jo AJ, Park CM, Kim HJ, Kim YJ, Park DW. Level of blood pressure control and cardiovascular events: SPRINT Criteria versus the 2014 Hypertension Recommendations. J Am Coll Cardiol 2016;67:2821-31.

22. Doubal FN, Ali M, Batty GD, Charidimou A, Eriksdotter M, Hofmann-Apitius $\mathrm{M}$, et al. Big data and data repurposing: using existing data to answer new questions in vascular dementia research. BMC Neurol 2017;17:72.

23. Amini L, Azarpazhouh R, Farzadfar MT, Mousavi SA, Jazaieri F, Khorvash F, et al. Prediction and control of stroke by data mining. Int J Prev Med 2013;4(Suppl 2):S245-9.

24. Li X, Liu H, Du X, Zhang P, Hu G, Xie G, et al. Integrated machine learning approaches for predicting ischemic stroke and thromboembolism in atrial fibrillation. AMIA Annu Symp Proc 2017;2016:799-807.

25. Arslan AK, Colak C, Sarihan ME. Different medical data 
mining approaches based prediction of ischemic stroke. Comput Methods Programs Biomed 2016;130:87-92.

26. Yoon S, Gutierrez J. Behavior correlates of post-stroke disability using data mining and infographics. $\mathrm{Br} \mathrm{J}$ Med Med Res 2016;11:BJMMR.21601.

27. Sporns O. Structure and function of complex brain networks. Dialogues Clin Neurosci 2013;15:247-62.

28. Auriat AM, Neva JL, Peters S, Ferris JK, Boyd LA. A review of transcranial magnetic stimulation and multimodal neuroimaging to characterize post-stroke neuroplasticity. Front Neurol 2015;6:226.

29. Kuceyeski A, Navi BB, Kamel H, Relkin N, Villanueva M, Raj A, et al. Exploring the brain's structural connectome: a quantitative stroke lesion-dysfunction mapping study. Hum Brain Mapp 2015;36:2147-60.

30. Weng SF, Reps J, Kai J, Garibaldi JM, Qureshi N. Can machine-learning improve cardiovascular risk prediction using routine clinical data? PLoS One 2017;12:e0174944.

31. Ong BT, Sugiura K, Zettsu K. Dynamically pre-trained deep recurrent neural networks using environmental monitoring data for predicting PM(2.5). Neural Comput Appl 2016;27:1553-66.

32. Labovitz DL, Shafner L, Reyes Gil M, Virmani D, Hanina A. Using artificial intelligence to reduce the risk of nonadherence in patients on anticoagulation therapy. Stroke 2017;48:1416-9.

33. Abedi V, Goyal N, Tsivgoulis G, Hosseinichimeh N, Hontecillas R, Bassaganya-Riera J, et al. Novel screening tool for stroke using artificial neural network. Stroke 2017;48: 1678-81.

34. Asadi H, Dowling R, Yan B, Mitchell P. Machine learning for outcome prediction of acute ischemic stroke post intra-arterial therapy. PLoS One 2014;9:e88225.

35. Wang S, Zhang Y, Zhan T, Phillips P, Zhang Y, Liu G, et al. Pathological brain detection by artificial intelligence in magnetic resonance imaging scanning. Prog Electromagn Res 2016;156:105-33.

36. Gladstone DJ, Spring M, Dorian P, Panzov V, Thorpe KE, Hall J, et al. Atrial fibrillation in patients with cryptogenic stroke. N Engl J Med 2014;370:2467-77.

37. Sanna T, Diener HC, Passman RS, Di Lazzaro V, Bernstein RA, Morillo CA, et al. Cryptogenic stroke and underlying atrial fibrillation. N Engl J Med 2014;370:2478-86.

38. Chang WH, Kim YH. Robot-assisted therapy in stroke rehabilitation. J Stroke 2013;15:174-81.

39. Chen G, Chan CK, Guo Z, Yu H. A review of lower extremity assistive robotic exoskeletons in rehabilitation thera- py. Crit Rev Biomed Eng 2013;41:343-63.

40. Awad LN, Bae J, O’Donnell K, De Rossi SMM, Hendron K, Sloot LH, et al. A soft robotic exosuit improves walking in patients after stroke. Sci Transl Med 2017;9:eaai9084.

41. Remsik A, Young B, Vermilyea R, Kiekhoefer L, Abrams J, Evander Elmore $\mathrm{S}$, et al. A review of the progression and future implications of brain-computer interface therapies for restoration of distal upper extremity motor function after stroke. Expert Rev Med Devices 2016;13:445-54.

42. Shih JJ, Krusienski DJ, Wolpaw JR. Brain-computer interfaces in medicine. Mayo Clin Proc 2012;87:268-79.

43. Bundy DT, Souders L, Baranyai K, Leonard L, Schalk G, Coker $\mathrm{R}$, et al. Contralesional brain-computer interface control of a powered exoskeleton for motor recovery in chronic stroke survivors. Stroke 2017;48:1908-15.

44. Tack P, Victor J, Gemmel P, Annemans L. 3D-printing techniques in a medical setting: a systematic literature review. Biomed Eng Online 2016;15:115.

45. Baronio G, Harran S, Signoroni A. A critical analysis of a hand orthosis reverse engineering and 3D printing process. Appl Bionics Biomech 2016;2016:8347478.

46. Ikram MA, Seshadri S, Bis JC, Fornage M, DeStefano AL, Aulchenko YS, et al. Genomewide association studies of stroke. N Engl J Med 2009;360:1718-28.

47. Khera AV, Emdin CA, Drake I, Natarajan P, Bick AG, Cook NR, et al. Genetic risk, adherence to a healthy lifestyle, and coronary disease. N Engl J Med 2016;375:2349-58.

48. Choi JC. Genetics of cerebral small vessel disease. J Stroke 2015;17:7-16.

49. Traylor M, Farrall M, Holliday EG, Sudlow C, Hopewell JC, Cheng YC, et al. Genetic risk factors for ischaemic stroke and its subtypes (the METASTROKE collaboration): a meta-analysis of genome-wide association studies. Lancet Neurol 2012;11:951-62.

50. Rostanski SK, Marshall RS. Precision medicine for ischemic stroke. JAMA Neurol 2016;73:773-4.

51. Fox CS, Hall JL, Arnett DK, Ashley EA, Delles C, Engler MB, et al. Future translational applications from the contemporary genomics era: a scientific statement from the American Heart Association. Circulation 2015;131:1715-36.

52. Cohen JC, Boerwinkle E, Mosley TH Jr, Hobbs HH. Sequence variations in PCSK9, low LDL, and protection against coronary heart disease. N Engl J Med 2006;354: 1264-72.

53. Stein EA, Mellis S, Yancopoulos GD, Stahl N, Logan D, Smith WB, et al. Effect of a monoclonal antibody to PCSK9 on LDL cholesterol. N Engl J Med 2012;366:1108- 
18.

54. Robinson JG, Farnier M, Krempf M, Bergeron J, Luc G, Averna $M$, et al. Efficacy and safety of alirocumab in reducing lipids and cardiovascular events. N Engl J Med 2015;372:1489-99.

55. Siironen J, Juvela S, Kanarek K, Vilkki J, Hernesniemi J, Lappalainen J. The Met allele of the BDNF Val66Met polymorphism predicts poor outcome among survivors of aneurysmal subarachnoid hemorrhage. Stroke 2007;38: 2858-60.

56. Kim JM, Stewart R, Park MS, Kang HJ, Kim SW, Shin IS, et al. Associations of BDNF genotype and promoter methylation with acute and long-term stroke outcomes in an East Asian cohort. PLoS One 2012;7:e51280.

57. Chang WH, Hwang JM, Uhm KE, Pascual-Leone A, Kim $\mathrm{YH}$. Corticospinal excitability in the non-dominant hand is affected by BDNF genotype. Neurol Sci 2017;38:241-7.

58. Cramer SC, Procaccio V; GAIN Americas; GAIN International Study Investigators. Correlation between genetic polymorphisms and stroke recovery: analysis of the GAIN Americas and GAIN International Studies. Eur J Neurol 2012;19:718-24.

59. Kim BR, Kim HY, Chun YI, Yun YM, Kim H, Choi DH, et al. Association between genetic variation in the dopamine system and motor recovery after stroke. Restor Neurol Neurosci 2016;34:925-34.

60. Uhm KE, Kim YH, Yoon KJ, Hwang JM, Chang WH. BDNF genotype influence the efficacy of rTMS in stroke patients. Neurosci Lett 2015;594:117-21.

61. Joly Y, Dyke SO, Knoppers BM, Pastinen T. Are data sharing and privacy protection mutually exclusive? Cell 2016; 167:1150-4.
62. Bang OY. Clinical trials of adult stem cell therapy in patients with ischemic stroke. J Clin Neurol 2016;12:14-20.

63. Bang OY, Kim EH, Cha JM, Moon GJ. Adult stem cell therapy for stroke: challenges and progress. J Stroke 2016;18: 256-66.

64. Hess DC, Wechsler LR, Clark WM, Savitz SI, Ford GA, Chiu $D$, et al. Safety and efficacy of multipotent adult progenitor cells in acute ischaemic stroke (MASTERS): a randomised, double-blind, placebo-controlled, phase 2 trial. Lancet Neurol 2017;16:360-8.

65. Kalladka D, Sinden J, Pollock K, Haig C, McLean J, Smith $W$, et al. Human neural stem cells in patients with chronic ischaemic stroke (PISCES): a phase 1, first-in-man study. Lancet 2016;388:787-96.

66. Kim SJ, Moon GJ, Chang WH, Kim YH, Bang OY; STARTING-2 (STem cell Application Researches and Trials In NeuroloGy-2) Collaborators. Intravenous transplantation of mesenchymal stem cells preconditioned with early phase stroke serum: current evidence and study protocol for a randomized trial. Trials 2013;14:317.

67. Kirouac DC, Zandstra PW. The systematic production of cells for cell therapies. Cell Stem Cell 2008;3:369-81.

68. Bensaid W, Triffitt JT, Blanchat C, Oudina K, Sedel L, Petite $\mathrm{H}$. A biodegradable fibrin scaffold for mesenchymal stem cell transplantation. Biomaterials 2003;24:2497502.

69. Joynt KE, Bhatt DL, Schwamm LH, Xian Y, Heidenreich PA, Fonarow GC, et al. Lack of impact of electronic health records on quality of care and outcomes for ischemic stroke. J Am Coll Cardiol 2015;65:1964-72.

70. Cabitza F, Rasoini R, Gensini GF. Unintended consequences of machine learning in medicine. JAMA 2017;318:517-8. 